\title{
Voltage Control Techniques for Electrical Distribution Networks Including Distributed Generation
}

\author{
T. Xu, P. C. Taylor \\ School of Engineering, University of Durham, UK \\ Tel: $0044-01913342469$; \\ E-mail: tao.xu2@durham.ac.uk,p.ctaylor@durham.ac.uk
}

\begin{abstract}
:
For the last three decades, a large scale integration of distributed generation (DG) is beginning to change the electrical distribution network from passive to active. Consequently, technical difficulties are created by significant impacts generated by DGs with voltage variation being the dominant effect. This paper presents a comprehensive review of voltage control techniques on electrical distribution networks connected with DG, recommendations are provided in terms of enhancing network voltage stability and maximising the DG utilisation.
\end{abstract}

Keywords: voltage control, renewable energy, distributed generation, electrical distribution network, active network management,

\section{INTRODUCTION}

The electric power distribution network has traditionally been operated in a passive mode where most of the electricity was generated by large power plants, delivered to the customers through distribution networks. Thus the flow of real and reactive power was from the higher to the lower voltage levels. Recently, in order to meet the requirement of the Kyoto protocol, distributed generation (DG) with renewable energy are expected to play an increasing role in emerging electric networks. The distribution network became an active system with power flows and voltages determined by the generation as well as the loads. In the last few years, this change was more perceptible due to the connections of various DG sources at the MV and LV levels. These sources include combined heat power (CHP) plants, hydro generators, wind plants and biomass units (Jenkins et al., 2000). In the near future, the DG on the networks will make a significant reduction of the total consumption of fossil-fuelled electricity, hence allowing a substantial minimisation of carbon dioxide emission (Liew and Strbac, 2002).

Distribution networks have not been designed to cope with power injections from DG, therefore the proliferation of DG on the electric networks results in a number of adverse impacts, including voltage variation, degraded protection, altered transient stability, bi-directional power flow and increased fault level, the voltage variation has been addressed as the dominant effect (Vovos et al. 2007). Typically, one of the most severe situation is that voltage magnitude at the proximity of DG exceeds the statutory limits during maximum power output from DG and minimum power demand from the network. Here the network experiences the largest reverse power flow and large voltage change which affects the network safety and stability.
Distribution network operators (DNOs) are responsible for operating the network within statutory limits. The voltage variation problem can be solved by either network, generator or load operational changes (utilizing the existing infrastructure) or network asset upgrades (Vovos et al., 2007). The network and generator operational changes, such as DG power curtailment, may conflict with contractual policies ('first on last off') between DNOs and DG. Whilst the network asset upgrades, such as reinforcement of networks, require significant investments on the distribution networks. DNOs need to justify the cost in terms of revenue benefit (Harrison and Wallace, 2005).

In the existing distribution networks with DG connected, intelligent/hybrid generator, load and network control, active network management become the main concerns in order to minimise the adverse impacts generated by the DG. Mitigation of voltage variation problems is no longer implemented in a centralized architecture, the controllers must move from $\mathrm{HV}$ to $\mathrm{MV}$ and LV levels, the control approach need to solve the problem locally in where it is generally applicable and commercial viable (ÓGorman and Redfern, 2004).

The aim of this paper is to provide a systematic technical review of voltage control techniques which have been employed on distribution networks with DG connected. It also presents further considerations for solving voltage variation issues in order to accommodate the growth of DG on the networks.

\section{VOLTAGE CONTROL IN ELECTRICAL DISTRIBUTION NETWORKS}

The voltage variation $\Delta V$ across the line can be approximated represented by the following equation:

$$
\Delta V=\frac{P R+Q X}{V}
$$


where, $\Delta V$ indicates voltage variation, $P$ and $Q$ represent active and reactive power output of DG, $X$ and $R$ are reactance and resistance of the line connecting to DG, $V$ is nominal voltage at the terminal of DG.

A simple radial feeder connected with a DG is shown in Fig.1. An on-load tap changer (OLTC) transformer, a local load, a reactive power compensator, an automatic voltage controllers (AVCs), a line drop compensator (LDC) and a energy storage device are also connected on the network.

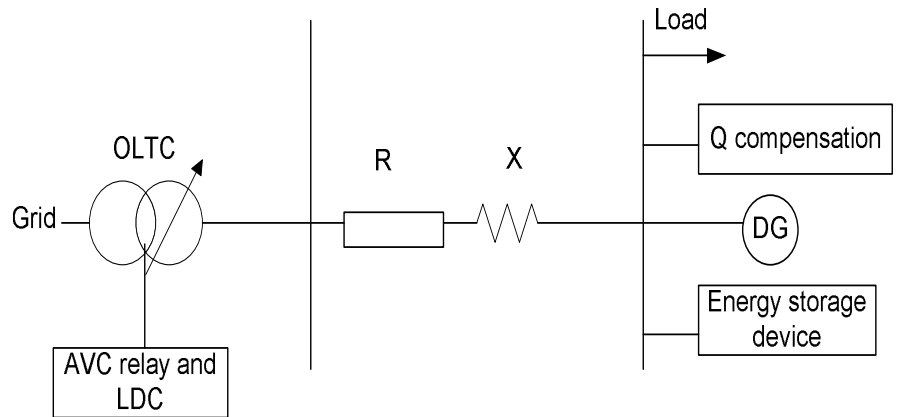

Fig. 1. Simple radial feeder with connected DG

Generally, compared with transmission line, the $X / R$ ratio is relatively low in a distribution network. According to Equation 1, any significant amount of power injected by DG will result in voltage rise/drop on the distribution network, especially in a weak distribution feeder with high impedance. The voltage variation would also depend on several factors including DG size and location, and method of voltage regulation. In the literature, extensive research has been undertaken to address this issue, and the following techniques have been successfully employed in a range of applications.

\subsection{On-load Tap Changers(OLTCs)}

The most common voltage control technique on the distribution network is to use OLTCs which maintain a stable secondary voltage by selecting the appropriate tap position. It is an effective way to control the voltage by shifting phase angle and adjusting voltage magnitude. It is usually in conjunction with AVC relay and LDC. The AVC relay continuously monitors the output voltage from the transformer, a tap change command will be initiated when the voltage is above the pre-set limits. The LDC is used to compensate additional voltage drop on the line between the transformer and load location, particularly, in the far end of the feeder.

An intentional time delay, normally within 30 to 60 seconds, is always implemented in OLTCs so as to avoid unnecessary tap change operations during the transient voltage fluctuations. The tap change operation usually takes 3-10 minutes to move from one position to another, and a several minute time interval between frequent operations is also required with considering the oxidation of tank oil (Tso et al., 1995).

Dai and Baghzouz (2003) showed that the coordination between DG outputs and OLTC tap controls is a necessity in order to allow higher DG integration. Otherwise, power injection levels can be severely limited if substation voltage is kept constant by the OLTC transformer.

Kim and Kim (2001) proposed an algorithm that can integrate $\mathrm{DG}$ on multiple feeders by using LDC without changing OLTC position. This method can minimize the voltage variation, prevent frequent OLTC operations, however it restricts the DG power integration.

In a DG connected network, Viawan et al. (2007) implemented OLTCs/LDCs/AVC relays on a MV feeder and a multiple MV feeder networks respectively. Simulations were carried out with and without DG connections, the results demonstrated that the use of OLTCs/LDCs/AVC relays can effectively minimise the voltage variation, also can significant increase the maximum size of DG that can be connected to a given feeder without disrupting voltage profile.

\subsection{Generator power factor control (PFC)}

By taking use of an AVC relay with DGs, synchronous generators are able to adjust their reactive power output to affect the busbar voltage. This operation could result in several severe problems, including high current and over heating, triggering the excitation limit or over current protection and disconnecting the generator from the network. In order to ensure the network safety, DGs are not permitted to use AVC to adjust their voltage. Therefore, PFC has been chosen by most DGs.

In PFC, $P / Q$ is maintained constant, according to Equation 1, any fluctuation in $P$ brings about proportional variation of voltage. If $Q$ can be compensated for the voltage variation generated by $P$ by adjusting in the opposite direction, then the voltage variation can be maintained within statutory limits (Vovos et al., 2007). For voltage rise situation, a more leading power factor is required at which the DG is to be connected.

Wallace and Kiprakis (2002) proposed a voltage control method for DG which assumed a more flexible directive from DNOs in terms of the voltage control by DG. The target was to develop a voltage control method capable of keeping DG online during light and/or heavy loading conditions by combining the advantages of AVC and PFC. This approach was also implemented to improve the steady state and slow transient voltage profile and increase energy dispatch.

\subsection{Power curtailment}

Currently, due to the inflexibility of the voltage control strategies, DNOs trip whole DG from the network to solve the voltage rise problem. This operation largely wastes the potential renewable energy and reduce the profit of DGs (Mogos and Guillaud, 2004). Therefore DG power output curtailment is proposed as a a straight forward method to solve voltage variation problems by reducing DG power production. However 'first on last off' agreement between the DG owners and DNOs adds complexity to the power curtailment technique.

This control strategy can be easily implemented in biomass, hydro and CHP plants. According to the stochastic operation mode of wind farm, the most effective way for 
power curtailment would be increase/decrease the speed of wind turbines by using pitch control.

Taking advantage of using reactive power control and real power curtailment, Mogos and Guillaud (2004) proposed a two mode switching voltage regulating method. Due to load variations on the network, the voltage may rise/drop beyond the admissible limits, the DG must begin to consume reactive power at first until the acceptable limits are reached. If the reactive power control is not sufficient to keep the voltage on the appropriate range, the control strategy will be switched to real power control to decrease/increase power production.

\subsection{Energy storage}

Kondoh et al. (2000) pointed out that energy storage devices including pumped hydro storage, compressed air energy storage (CAES), hydrogen, lead acid batteries, superconducting magnetic energy storage (SMES), flywheel and capacitors are expected to be wide spread in DG connected networks. Currently, energy storage technologies are at various stages of development and deployment. Pumped hydro and lead acid batteries are the most widespread storage technology deployed on power systems, they are technically and commercially mature. Whilst superconducting magnetic energy storage is technically possible but is not mature.

Energy storage devices have been recognised as an environmentally benign means of modulating renewable generation and providing reserves. These devices use a power conversion system (PCS) to connect to the distribution system, they can source or sink both active and reactive power to compensate for voltage variations in the short or medium term. For longer durations of voltage problems, excessive energy storage capability is required with a high capital cost (Choi and Kim, 2000b). The PCS cost is usually a significant proportion of the overall cost of an energy storage facility. As this cost is predominantly current driven, the chosen objective is to minimise the PCS current required by the network to maintain the minimum voltage limit.

Wind power generators have gained increased operational benefits and economic returns by combining energy storage devices (Lund and Paatero, 2006). Energy storage technologies can store the surplus during the periods when wind generation exceeds the demand and then be used to cover periods when the load is greater than the generation. Lund and Paatero (2006) demonstrated that approximately $1 \mathrm{MWh}$ storage per MW of wind power is enough to reduce at least $10 \%$ of the local voltage rise in weak networks.

\subsection{Network reconfiguration}

Network reconfiguration refers to the process of closing/opening the normal open point (NOP) between two radial feeders to form the 'ring' operation. Full utilisation of network resources and minimisation of system losses are benefits of this control strategy. This technique has been widely used for network loss reduction and load balance (Celli et al., 2005; Choi and Kim, 2000a; Aoki et al., 1998). Artificial intelligence techniques, such as, fuzzy logic and genetic algorithm (GA) have been applied to maximize load ability margin and minimise system losses.

Venkatesh et al. (2004) presented a novel solution by using fuzzy adaptation of the evolutionary programming technique (FEP) for optimal reconfiguration. It uses fuzzy modelling methods to model the two objectives of loadability margin maximization and obtaining the best voltage profile.

However this technique is a new topic in voltage control on DG connected networks. Owing to the network complexity, there are several issues that need to be taken into account in the design:

- How to choose the best location of NOPs on the network;

- How to cooperate with existing network restoration strategies;

- How to decide the operating sequences when multiple operations are undertaken on the network;

- How to cooperate with other voltage control techniques.

\subsection{Static synchronous compensator(STATCOM)}

A STATCOM is a flexible AC transmission systems (FACTS)device, it is a voltage-source converter based device which converts a $\mathrm{DC}$ input voltage into an $\mathrm{AC}$ output voltage in order to compensate the reactive power of the system. Usually the reactive output of a STATCOM is regulated to maintain the desired $\mathrm{AC}$ voltage at the bus, to which a STATCOM is connected. It can provide voltage control in either transmission or distribution system with a fast control response. The function is similar to reactive power control of the generation, except that a STATCOM provides a solution that is independent of the generator. Currently, the deployment of STATCOM is restricted by high costs.

Due to the fast response of STATCOM, modern control strategies, such as linear quadratic regulator (LQR), can be provided for voltage control. Rao et al. (2000) implemented PI, pole-placement and LQR controllers on the STATCOM respectively, the performances were compared in terms of response profile and control effort. The simulation results showed that the PI and LQR controller exhibited comparable responses. At extreme loading cases, however, the LQR controller had superior robustness. The proposed control methodologies were applied on a STATCOM in a traditional radial feeder, they can be easily extended to a network connected with multiple DGs.

\subsection{Demand side management (DSM)}

DSM refers to cooperative activities between the DNOs and their customers to implement options for increasing the efficiency of energy utilization, with resulting benefits to the customer, DNOs, and society as a whole. DSM is used to temporarily reduce the total power consumption increase, hence maintaining network safety and stability, maximising energy efficiency. This technique has been increasingly employed on LV networks (Fretheim, 2003).

The requirement for DSM applications is that the loads can be controlled by DNOs, and agreed to be modulated 
when necessary, it convinced to use more energy during off peak hours.

A DSM system consisting a central controller, and four load controllers has been applied on a $11 k V$ distribution network with a $2.6 \mathrm{MW}$ wind power generator (Seng and Taylor, 2006). Each load controller governs a balanced three phase load at $415 \mathrm{~V}$ network, The central controller monitors the voltage on the $415 \mathrm{~V}$ network, the load controller will switch in its load as soon as the voltage is greater than the pre-set limits. This work demonstrated that the advantages of using DSM can be used to mitigate voltage variation problems with minimum network reinforcement and minimum constraint of power output of DGs.

\subsection{Hybrid and cooperative control methodologies}

Due to the complexity of the existing DG connected networks, a single control strategy is often insufficient in solving complex voltage problems. Therefore, hybrid and cooperative methodologies are widely employed, compared with single control strategy. Hybrid and cooperative approaches manage different aspects and various situations on the network, State estimation and overall decision making strategies play a vital role in the overall system.

A simple but practical control strategy has been designed by Hird et al. (2003). It concentrated on designing a voltage controller that controls the AVC relay in a $33 / 11 k \mathrm{~V}$ primary substation in the UK. The controller featured a statistical state estimation algorithm with a control strategy. The state estimation algorithm calculates the expected value and standard deviation of the voltage magnitude at the each node on the network by utilising weighted least square algorithm and Newton Raphson method. The control strategy need to compare the voltage magnitude estimations with the DNO's acceptable range, if an estimate drops outside the range, a tap change operation will be issued by altering the AVC target voltage.

In Japan, integrated automatic OLTCs with LDCs are called step voltage regulators (SVRs). In order to avoid frequent tap changing, SVR has a dead band value for a target control voltage and time delay from a few seconds to a few minutes. However, when the DGs connect on the network, they may reach full power output from zero output within one minute, in which SVRs cannot take appropriate operation because of the time delay.

Unified power flow controller (UPFC) is one of the FACTS devices to regulate the line voltage. Because of the fast response, this equipment can be effectively used to protect against rapid voltage fluctuations within the prescribed voltage ranges. However, for slow voltage variations, it could be over compensated.

Naka et al. (2001) combined SVR and UPFC as a cooperative control solution for voltage regulation. When a large power injection occurred on the network, UPFC is utilised to compensate the rapid voltage fluctuations, then the reference voltage value of the parallel part of UPFC is gradually corrected and the compensation amount by UPFC is gradually decreased. The voltage reference value for the serial part of UPFC is constant. Therefore, SVR can be employed to compensate the parallel part of UPFC by changing the tap position. The UPFC is operating in a standby mode to wait for the next rapid voltage fluctuation.

\section{FURTHER CONSIDERATIONS OF VOLTAGE CONTROL TECHNIQUES ON ELECTRICAL DISTRIBUTION NETWORKS INCLUDING DG}

Electric network management system is in the era of innovation, traditionally, the network is operated with pre-set control strategies to meet the forecast load. In the future, in order to achieve more reliable and efficient performance, distribution systems will increasingly rely on technologies which actively shape the end-users response. Therefore network management system will be operated in an automated feedback mode with feedback information from DG, consumers and other distributed actors. Ideally, the network will be maintained completely active without pre-programmed operations (Ilic et al., 2007).

Consequently, in order to cope with dramatic changes on network management system, intelligent distributed controllers will be widespread on the network to minimise the voltage impacts. The control structure will move from simple control strategy to two hierarchical level operation. The fundamental level is local level and the second level is coordinated level. The local voltage control aims to maintain voltage at DG units in a fast control response. The coordinated level considers a system wide perspective for voltage control of a distribution network. It needs to tackle multiple generation schemes, multiple types of interrelated control actions, multiple and possibly conflicting criteria and multiple network topologies and configurations. The real time voltage control operation relies on online decisions in response to the varying system conditions.

This hierarchical control structure intends to remove the current 'fit and forget' DG connection policy, make the best use of DG energy with minimal voltage variations. More automated adaptation strategies are expected to minimize the adverse DG impacts and cope with the versatile demand requirement on the networks. Power electronics equipment, such as STATCOM and UPFC, and high efficiency communication techniques will be widely implemented on the networks in order to improve the control response.

\section{CONCLUSION}

Controlling the voltage on a distribution network with $\mathrm{DG}$ is an important and challenging issue for the DNOs, DG owners and load customers. With increasing DG connections on the networks, this issue is becoming more complex. Since each existing control technique has its advantages and drawbacks, an ideal solution is to employ different techniques in different scenarios with the best balance between cost and technical impacts. Intelligent and practical voltage control techniques associated with active network management systems can increase the level of DG penetration with maximum utilisation of the existing network. 


\section{REFERENCES}

K. Aoki, H. Kawabara, T. Satoh, and M. Kanezashi. An efficient algorithm for load balancing of transformers and feeders. IEEE Transactions on Power Delivery, 3 (4):1865-1872, 1998

G. Celli, M. Loddo, F. Pilo, and A. Abur. On-line network reconfiguration for loss reduction in distribution networks with distributed generation. CIRED $18^{t} h$ International Conference on Electricity Distribution, 2005.

J H. Choi and J C. Kim. Network reconfiguration at the power distribution system with dispersed generations for loss reduction. Proc. IEEE Power Engineering Society Winter Meeting, 4:2363-2367, 2000a.

J H. Choi and J K. Kim. Advanced voltage regulation method at the power distribution systems interconnected with dispersed storage and generation systems. IEEE Trans. on Power Delivery, 15(2):691-696, 2000b.

C. Dai and Y. Baghzouz. On the voltage profile of distribution feeders with distributed generation. Proc. IEEE Power Engineering Society Summer Meeting, 1: 456-461, July 2003.

S. Fretheim. Technology for demand side management and demand side bidding for grid companies. CIRED $17^{t} h$ International Conference on Electricity Distribution, May 2003.

G P. Harrison and A R. Wallace. Maximising renewable energy intergartion within electrical networks. World Renewable Energy Congress (WREC2005), May 2005.

M. Hird, N. Jenkins, and P C. Taylor. An active $11 \mathrm{kv}$ voltage controller: Practical considerations. CIRED $17^{t} h$ International Conference on Electricity Distribution, May 2003.

M. Ilic, J W. Black, and M. Prica. Distributed electric power systems of the future: Institutional and technological drivers for near-optimal performance. Electric Power Systems Research, (77):11601177, 2007.

N. Jenkins, R. Allan, P. Crossley, D. Kirschen, and G. Strbac. Embedded Generation. IEE, UK, 2000.

T E. Kim and J E. Kim. A method for determining the introduction limit of distributed generation in distribution system. Proc. IEEE Power Engineering Society Summer Meeting, 1:456-461, July 2001.

J. Kondoh, I. Ishii, H. Yamaguchi, A. Murata, K. Otani, K. Sakuta, N. Higuchi, S. Sekine, and M. Kamimoto. Electrical energy storage systems for energy networks. Energy Conversion and Management, (41):1863-1874, 2000.

S N Liew and G. Strbac. Maximising penetration of wind generation in existing distribution networks. IEE Proc. Generation, Transmission and Distribution, 149(3):256$262,2002$.

P D. Lund and J V. Paatero. Energy storage options for improving wind power quality. Nordic Wind Power Conference, May 2006.

E F. Mogos and X. Guillaud. A voltage regulation system for distributed generation. IEEE Power Systems Conference and Exposition, 2:787-794, October 2004.

S. Naka, T. Genji, T. Yura, S. Takayama, H. Tokuda, and Y. Fukuyama. Cooperative control method for voltage control equipment considering interconnection of distributed generators. Proc. of ICEE Conference, July 2001.
R. ÓGorman and M A. Redfern. Voltage control problems on modern distribution systems. IEEE Power Engineering Society General Meeting, 1:662-667, June 2004.

P. Rao, M L. Crow, and Z. Yang. Statcom control for power system voltage control applications. IEEE Transactions On Power Delivery, 15(4), October 2000.

L Y. Seng and P C. Taylor. Innovative application of demand side management to power systems. International Conference on Industrial and Information Systems, August 2006.

S K. Tso, T X. Zhu, Q Y. Zeng, and K L. Lo. Physical simulation study of dynamic voltage instability (in power systems). International Conference on Energy Management and Power Delivery, 1:348-353, 1995.

B. Venkatesh, R. Ranjan, and H B. Gooi. Optimal reconfiguration of radial distribution systems to maximize loadability. IEEE Transactions On Power Systems, 19 (1), February 2004.

F A. Viawan, A. Sannino, and J. Daalder. Voltage control with on-load tap changers in medium voltage feeders in presence of distributed generation. Electric Power System Research, (77):1314-1322, 2007.

P N. Vovos, A E. Kirprakis, A R. Wallace, and G P. Harrison. Centralised and distributed voltage control: Impact on distributed generation penetration. IEEE Trans. on Power Systems, 22(1):476-482, February 2007.

A R. Wallace and A E. Kiprakis. Reduction of voltage violations from embedded generators connected to the distribution network by intelligent reactive power control. Proc. IEE $5^{t} h$ International Conference on Power System Management and Control PSMC, April 2002. 